\title{
Broad iron emission lines in Seyfert galaxies - re-condensation of gas onto an inner disk below the ADAF?
}

\author{
E. Meyer-Hofmeister ${ }^{1}$ and F. Meyer ${ }^{1}$
}

\begin{abstract}
Max-Planck-Institut für Astrophysik, Karl- Schwarzschild-Str. 1, 85740 Garching, Germany
e-mail: emm@mpa-garching.mpg.de
\end{abstract}

Received 27 July 2010 / Accepted 5 December 2010

\begin{abstract}
Context. The number of strong iron $K_{\alpha}$ line detections in Seyfert AGN is clearly growing in the Chandra, XMM-Newton and Suzaku era. The iron emission lines are broad, some are relativistically blurred. These relativistic disk lines have also been observed for galactic black hole X-ray binaries. Thermal components found in hard spectra were interpreted as an indication for a weak inner cool accretion disk underneath a hot corona.

Aims. Accretion in low-mass X-ray binaries (LMXB) occurs during phases of high and low mass accretion rate, outburst and quiescence, soft and hard spectral state, respectively. After the soft/hard transition for some sources a thermal component is found, which can be interpreted as sustained by re-condensation of gas from an advection-dominated flow (ADAF) onto the disk. In view of the similarity of accretion flows around stellar mass and supermassive black holes we discuss whether the broad iron emission lines in Seyfert 1 AGN (active galactic nuclei) can be understood as arising from a similar accretion flow geometry as in X-ray binaries. Methods. We derive accretion rates for those Seyfert galaxies for which broad iron emission lines were observed, the "best candidates" in the investigations of Miller (2007, ARA\&A, 45, 441) and Nandra et al. (2007, MNRAS, 382, 194). For the evaluation of the Eddington-scaled rates we use the observed X-ray luminosity, bolometric corrections and black hole masses from the literature.

Results. The accretion rates derived for the Seyfert galaxies in our sample are less than 0.1 of the Eddington rate for more than half of the sources. For $10^{7}$ to $10^{8} M_{\odot}$ black holes in Seyfert 1 AGN this limit corresponds to 0.01 to $0.2 M_{\odot} / y r$. This documents that the sources probably are in a hard spectral state and iron emission lines can arise from an inner weak accretion disk surrounded by an $\mathrm{ADAF}$ as predicted by the re-condensation model. Some of the remaining sources with higher accretion rates may be in a spectral state that is comparable to the "very high" state of LMXBs.

Conclusions. Our investigation shows that in quite a number of Seyfert AGN the broad iron emission lines may indeed originate in a weak inner disk below the ADAF, close to the black hole, indicating the same accretion flow geometry as recently found for LMXBs. For the accretion history one then concludes that the accretion rates were higher in the outer radii at some earlier time.
\end{abstract}

Key words. accretion, accretion disks - X-rays: galaxies - black hole physics - galaxies: Seyfert - galaxies: individual: MCG-6-30-15 - galaxies: individual: 1H 0707-495

\section{Introduction}

The physical processes of accretion onto galactic and supermassive black holes and the accretion flow geometry in the innermost regions are key features for modeling relativistic spectral lines and for estimates of the black hole spin. Observations in recent years with the Chandra X-ray Observatory, the X-ray MultiMirror Mission-Newton and Suzaku reveal for some AGN broad, often relativistic iron emission lines from the innermost regions close to the black hole. Iron emission lines are the most obvious response of an accretion disk to irradiation by an external source of hard X-rays. When X-rays from a hot coronal flow fall on optically thick cool material, they induce fluorescence and are backscattered, resulting in a Compton reflection spectrum with a prominent emission line of iron $\mathrm{K} \alpha$. The accretion geometry strongly depends on the mass accretion rate. For LMXBs it is well known that on the one hand for high rates an optically thick, geometrically thin, radiatively efficient Shakura-Sunyaev accretion disk reaches inward to the innermost stable circular orbit (ISCO), or on the other hand for low accretion rates (lower than the critical rate of spectral state transition $\dot{m}_{\text {crit }}$ ), a more spherical optically thin, hot advection-dominated flow fills the inner region (ADAF; Narayan \& Yi 1994, 1995; Abramowicz et al. 1995; see Narayan 2005; Yuan 2007; Narayan \& McClintock 2008, for reviews). At larger distance an outer Shakura-Sunyaev disk exists, whose truncation radius recedes as the mass accretion rate decreases. These "high" and "low" states are documented by a soft and hard spectrum. The critical accretion rate $\dot{m}_{\text {crit }}$ lies around a few to 10 percent of the Eddington accretion rate (Esin et al. 1997).

For supermassive black holes, especially low-luminosity AGN, truncated thin disks were found, along with a hard spectrum (Narayan et al. 1998). The power-law spectrum arising from the coronal emission is scale-invariant (accretion rate in units of Eddington accretion rate), while the disk emission of AGN peaks in the UV range, in contrast to the emission in soft $\mathrm{X}$-rays from disks around stellar mass black holes. Vasudevan \& Fabian (2007) discuss the similarity of accretion states in AGN and galactic black hole sources and point out that the radiation in the UV is an important contribution to the bolometric luminosity. They derived bolometric corrections for AGN using recent results from the Far Ultraviolet Spectroscopic Explorer (FUSE), together with data from the Optical Monitor (OM) archives and $\mathrm{X}$-ray data from the XMM-Newton archive. They expanded the 


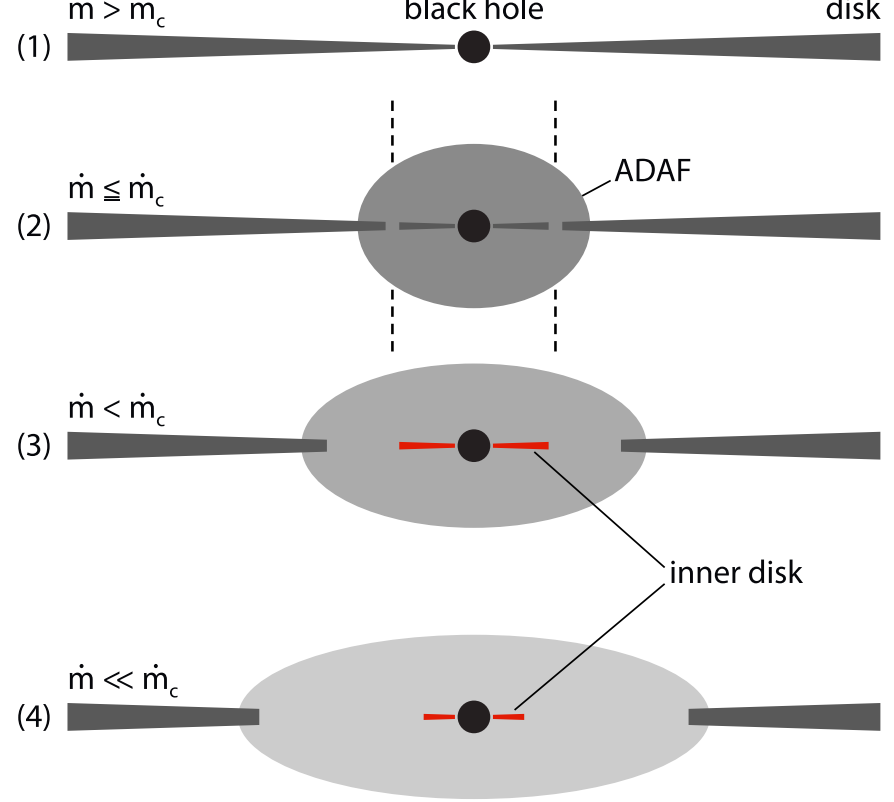

Fig. 1. Change of the geometry of the accretion flow with decreasing mass accretion rate scaled to the Eddington rate $\dot{m},\left(\dot{m}_{\mathrm{c}}\right.$ critical rate for which the state transition happens): (1) soft state, (2) transition to the hard state begins, formation of a gap where evaporation is most effective (3-4) hard states with disk truncation receding outward, the weak inner disk disappears (Fig. 1, Meyer-Hofmeister et al. 2009).

investigation and for the first time took into account simultaneous observations (Vasudevan \& Fabian 2009).

The accretion geometry in the two spectral states, either an ADAF (or one of its variants) in the inner regions or a disk reaching inward to the ISCO, seemed to exclude each other for a long time. But the recent observations of LMXBs seem to indicate that during in intermediate state, the brightest hard state (after soft/hard transition in outburst decay), an ADAF in the inner region and a weak innermost disk can both be present at the same time (see Fig. 1). These co-existing hot and cool gas flows inward toward the black hole clearly interact. The interaction causes mass and angular momentum exchange between disk and ADAF, either a mass flow from the disk into the ADAF, evaporation of the disk, or a reverse mass flow, re-condensation of gas from the ADAF onto the disk (Liu et al. 2006; Meyer et al. 2007).

Indications for a disk near the black hole during a canonical low-hard state were found for several X-ray binaries, with clearest hints in GX 339-4, SWIFT J1753.5-0127 and XTE J1817330. The re-condensation model allows us to understand the observed mass flow rates in the inner disk (Liu et al. 2007; Taam et al. 2008). Observational evidence for the possible presence of a thermal component during the hard state in eight sources, broad skewed $\mathrm{Fe} \mathrm{K} \alpha$ lines in half of the sample, were shown in the recent work by Reis et al. (2010). The authors interprete the reflection features as caused by illumination of a more-or-less permanent disk by the hard, power-law component of a jet.

We note that an extremely skewed relativistic Fe K $\alpha$ emission line was also found in the spectrum of GX 339-4 during the bright phase of its 2002-2003 outburst, in a "very high" state. As Miller (2007) pointed out, this observation is of interest for understanding the relativistic iron lines observed in Seyfert 1 AGN. We discuss these observations in connection with the detection of broad iron $\mathrm{K}$ and $\mathrm{L}$ line emission in the narrow-line galaxy 1H0707-495.
The existence (at least for some time) of a weak disk in the innermost region around stellar mass black holes during the hard spectral state strengthens the expectation to find the same phenomenon for accreting supermassive black holes as well. Seyfert 1 AGN are the class of supermassive black holes that offer the best view of the innermost accretion region. Tanaka et al. (1995) observed the first asymmetric disk line profile in the Seyfert 1 AGN MCG-6-30-15 using the ASCA/SIS. Now XMM-Newton, Chandra and Suzaku allow us to detect and measure relativistic disk lines in Seyferts. The Fe K-shell emission lines are the strongest X-ray emission lines both in AGN and Xray binaries. In a detailed review of relativistic X-ray emission lines from inner accretion disks around black holes Miller (2007) discusses observational and theoretical developments. The review of Nandra et al. (2007) focuses on broad iron lines in Seyfert galaxies observed by XMM-Newton and raises the question how frequent this broadening is, whether it originates in an accretion disk, and how robust the evidence for an accretion disk is. At first glance it is not clear whether the lines originate in a weak disk below a prominent hard coronal X-ray flux or are connected with accretion via an untruncated disk during the "very high" state.

The aim of our paper is to analyze under which circumstances the broad iron emission lines in Seyfert galaxies may originate in a weak disk in the innermost region around the black hole, similar to the situation of stellar mass black holes (and neutron stars). In Sect. 2 we refer to the process of disk evaporation, which for low accretion rates leads to the truncation of the standard Shakura-Sunyaev disk at a certain radius. In Sect. 3 we discuss how changes of the mass flow rate result in distinct spectral states for both LMXBs and AGN. The mass flow toward the inner regions can be modulated by the ionization instability and magnetorotational and gravitational instabilities (Siemiginowska et al. 1996; Menou \& Quataert 2001). In the Appendix we discuss how the ionization instability can modulate the mass flow toward the inner regions. As a consequence of the disk evaporation process a new picture arises for the presence of these disk instabilities.

These mass flow variations can cause changes between hard and soft spectral states and lead to the temporary existence of a weak inner disk. We discuss in Sect. 4 for which rates the appearance of an inner disk can be expected as a transient phenomenon in Seyfert galaxies. In Sect. 5 we derive accretion rates from observations for the best candidates of broad iron emission lines in the samples of Seyfert galaxies of Miller (2007) and Nandra et al. (2007). In Sect. 6 we discuss a different accretion flow geometry: broad emission lines from an untruncated disk in bright sources in the "very high" state.

We note that a different picture is also discussed for the accretion flow geometry, an always present accretion disk together with a jet. For supermassive black holes, Sgr A* and lowluminosity AGN such a jet-dominated situation was suggested (Falcke \& Biermann 1999; Falcke \& Markoff 2000; Falcke et al. 2000 ) as an alternative to the ADAF solution (Narayan et al. 1998; Di Matteo et al. 2000, 2003). The iron emission lines could be caused by illumination from a non-thermal jet.

\section{Evaporation leading to a truncated disk}

The process of evaporation of matter from an accretion disk via a siphon flow to a hot corona/ADAF was generally proposed for disks surrounding a compact object (Meyer \& Meyer-Hofmeister 1994). For the interaction between accretion disk and corona/ADAF around compact objects of different mass 
the physics is the same from neutron stars and black holes in LMXBs to supermassive black holes in AGN (Meyer et al. 2000; Liu et al. 2002). It is always the interaction between a coronal hot gas and a cool disk below: frictional heat released in the corona flows down into cooler and denser transition layers; there it is radiated away if the density is sufficiently high; if the density is too low, cool matter is heated and evaporates into the corona until an equilibrium density is established. Compton cooling adds to thermal conduction (Liu et al. 2007). Modeling allows us to investigate the effects of the chosen parameters, e.g. the viscosity value, or the inclusion of a magnetic pressure (Qiao \& Liu 2010) and to take into account hard and soft irradiation. Exact values for disk truncation and transition luminosity are difficult to obtain because of this freedom in the parameter choice and the limitations of a one-zone model used for describing the complex situation.

The surface temperatures in the disks around massive black holes do not change the situation, as was questioned in the work by McHardy (2009), because disk surface temperatures are much below the temperatures of the decisive radiating layers at the bottom of the coronae. Evaporation and disk truncation in AGN were investigated by Liu \& Meyer-Hofmeister (2001). In recent work Liu \& Taam (2009) suggest that the existence/nonexistence of the so-called broad line region of type 1 AGN is related to disk truncation.

\section{Distinct spectral states of LMXBs - analogous situation in AGN?}

For LMXBs a wealth of information on spectral states and transitions between them is available, because of the short timescales (shorter than for AGN - the dynamical time scales with the central mass, a factor of $10^{6}$ to $10^{8}$ difference) and the large number of objects in our vicinity. Chen et al. (1997) collected all available data at that time, 66 recorded outbursts already. The two review papers of McClintock \& Remillard (2006) and Remillard $\&$ McClintock (2006) give a compilation of the large number of observations and their theoretical interpretations. Accretion flows in black hole X-ray binaries and neutron star binaries are investigated by Done et al. (2007). The recent study by Dunn et al. (2010) includes additional recent observations.

What is the situation for AGN? Theoretically we expect the same properties of the accretion flow, distinct states in response to changes of the mass accretion rate, for all compact objects of different mass (Narayan et al. 1998). Observations for AGN seem to confirm this situation. In analogy to LMXBs in hard spectral state, low-luminosity accretion and truncated disks were found for AGN already many years ago (Narayan 2005). Ho (2009a) recently concluded that the massive black holes in most nearby galaxies reside in a low or quiescent state.

For LMXBs we know the clear change of spectra during the hard/soft and soft/hard transitions of Cyg X-1 in 1996 (Esin et al. 1998), the change of the photon index $\Gamma$. But for AGN the situation is not as clear, we can only draw some parallels from average spectral energy distributions. Vasudevan \& Fabian (2007) compared the slopes in the spectral energy distribution for sources in the ranges $0.0012<L_{\text {bol }} / L_{\text {Edd }}<0.032$ and $0.27<L_{\mathrm{bol}} / L_{\text {Edd }}<2.7$ and found a harder spectral shape for the lower luminosities, and softer X-ray spectra for the higher luminosities. Done \& Gierliński (2005) argued that complex absorption from a disk wind might affect the spectra, so that these could appear as being harder. Shemmer et al. (2006) studied the hard $\mathrm{X}$-ray spectral slope as an accretion rate indicator for luminous
AGN and found a dependence on the accretion rate for their sample.

Similarity between accretion in galactic and supermassive black holes is also found by comparing X-ray and optical variability. Measuring the X-ray variability power spectral density (PSD) of six Seyfert 1 galaxies Markowitz et al. (2003) pointed out a physical similarity with X-ray binaries. But Uttley \& McHardy (2005) found, e.g. for NGC 3227, an intrinsically hard $\mathrm{X}$-ray spectrum (photon index $\Gamma \sim 1.6$ ), yet a broad-band X-ray variability PSD reminiscent of black hole X-ray binaries in the high/soft state, and argued that the current nomenclature for the various states may be inappropriate. More Seyfert galaxies were investigated by Uttley \& McHardy (2005), McHardy et al. (2005) and Arévalo et al. (2006).

Because mass flow changes are a basic element in the consideration of spectral states in AGN as well, we add to our analysis a discussion of disk instabilities in the Appendix, which can produce these changes and trigger spectral transitions. We show that a new picture of the possible importance of the ionization instability arises from the truncation of inner disk regions, which can lead to an elimination of the instability. But the instability is expected to be present for black hole masses of $10^{7} M_{\odot}$ to about $10^{8} M_{\odot}$.

\section{A cool disk in the innermost region?}

\subsection{The re-condensation model}

Observations of LMXBs indicate cool gas in the neighborhood of the accreting black hole. Most observations were made at a time shortly after the soft/hard spectral state transition, which points to an intermediate state connected with the transition process.

During phases of decreasing mass flow rate, as in outburst decline, a gap opens between the outer disk and the inner region at the distance where the evaporation is most efficient, which is filled by an ADAF (Fig. 1). Within the short diffusion time the inner disk would disappear, if it were not sustained by a continuous supply of gas from the ADAF above. The observations indicate that the inner disk can survive. The re-condensation model (Liu et al. 2006; Meyer et al. 2007) allows us to understand the interaction: the ADAF is affected by thermal conduction to a cool accretion disk underneath. Such a model takes into account the exchange of energy and mass between the tenuous, inefficiently radiating, hot two-temperature corona and the uppermost layers of the disk. The re-condensation rate becomes a function of the mass flow rate in the ADAF, $\dot{m}_{\mathrm{ADAF}}$ (in Eddington accretion rate units), and the ratio of the distance to the ISCO and the distance of the outer edge of the inner disk, $R_{\mathrm{c}}$, which depends on $\dot{m}_{\mathrm{ADAF}}$.

$$
\dot{m}_{\mathrm{cond}} / \dot{m}_{\mathrm{ADAF}}=3.23 \times 10^{-3} \alpha^{-7} \dot{m}_{\mathrm{ADAF}}^{2} f\left(R_{\mathrm{ISCO}} / R_{\mathrm{c}}\right)
$$

with the function

$$
f(x)=1-6 x^{1 / 2}+5 x^{3 / 5} .
$$

The process works best at distances from 30 to 100 Schwarzschild radii, and suitable condensation rates are typically $10 \%$ of $\dot{m}_{\mathrm{ADAF}}$ (strongly dependent on the viscosity parameter $\alpha$ ). All condensated gas flows inward to the ISCO. As $\dot{m}_{\mathrm{ADAF}}$ decreases, the inner disk becomes smaller and the recondensation rate decreases. This inner disk can exist for a long time during the low/hard state, as long as the accretion rate is not too low, $\leq \dot{m} \approx 10^{-3}$ (Taam et al. 2008), and could still be present during the rise to the next outburst. 
The model was applied to X-ray observations of the black hole sources GX 339-4 and SWIFT J1753 and is able to explain the effective temperature of the thermal component (Liu et al. 2007). Compton cooling was found to be an important element in the re-condensation process (Taam et al. 2008).

The physics of the re-condensation process is the same for stellar mass and supermassive black holes. The disks in AGN have lower temperatures, thermal conduction occurs as in LMXB disks. The diffusion time for AGN disks close to the black hole is also short compared to the time within which the mass flow rate from the outer regions can change. This means that re-condensation is needed in AGN as well to sustain a cool innermost disk underneath the ADAF.

\subsection{Cool disks around stellar mass black holes. The observational evidence}

For investigating inner disks in AGN, the observations of stellarmass black hole sources are of interest. Besides the mostly cited observations of GX 339-4 and SWIFT J1753.5-0127 (Miller et al. 2006a,b; Reis et al. 2008; Tomsick et al. 2008), indications for the presence of weak inner disks are also found in several other sources (for the strength of the thermal components see Meyer-Hofmeister 2009), very recently also in the new X-ray transient XTE J1652-453 (Hiemstra et al. 2011). These thermal components were found in connection with outbursts, in outburst decline, usually during the brightest phases of the hard state (with the exception of Cyg X-1). Recently Reis et al. (2010) presented a study of eight stellar-mass black hole sources in the low/hard state and confirmed the existence of a thermal component for all of them, with iron $\mathrm{K} \alpha$ line emission in half of the sources. All observations of thermal components close to the ISCO document the presence of cool material close to the black hole (i.e. for dynamical reasons an inner disk) during the hard state. Unfortunately we cannot yet derive from this whether such an inner disk continues all the way out or if a gap exists at the distance where (according to the modeling) evaporation is most efficient.

Interestingly, a skewed relativistic $\mathrm{Fe} \mathrm{K} \alpha$ emission line was also observed for XTE J1650-500 and for GX 339-4 in "very high "state (Miller et al. 2002b, 2004), probably originating in a different accretion flow geometry for a high accretion rate, an untruncated disk in a very bright state.

\subsection{Mass flow rates appropriate for inner disks around supermassive black holes}

For AGN the situation should be similar to that for stellar mass black holes (for a definition of states see Remillard \& McClintock 2006): (1) a low/hard state, an ADAF in the inner region, accretion rates below a few percent to $0.1 \dot{M}_{\text {Eddington }}$ (spectrum hard); (2) a standard high/soft state (="thermal"), disk inward to the ISCO, accretion rates about 0.05 to $0.5 \dot{M}_{\text {Eddington }}$ (spectrum soft); (3) a very high state ("steep power law"), disk inward to the ISCO and an additional coronal layer above the disk, accretion rates just sub-Eddington (spectrum soft + hard component). During a decrease from the high/soft state to the low/hard state for some time a weak inner disk can be present (spectrum hard + weak thermal component).

We here focus on the situation shortly after the transition from the soft to the hard state, with an additional weak inner disk, as a relict of a former disk that reached inward to the ISCO in a preceding high/soft state. This means the accretion rates should lie between $\dot{m}_{\text {crit }} \approx 0.05$ to 0.1 and about $\frac{1}{10}$ of this rate (based on the evidence of such inner disks in LMXBs). This predicts accretion rates appropriate to allow inner disks in Seyfert 1 galaxies of $0.1 M_{\odot} /$ yr for a $10^{8} M_{\odot}$ black hole, one tenth of this rate for a $10^{7} M_{\odot}$ black holes (in our discussion of mass flow rate changes caused by the ionization instability these rates correspond to the dashed lines in Fig. A.1, Appendix).

\section{Broad iron lines in Seyfert 1 galaxies as indicators of an inner accretion disk}

\subsection{Observations}

Our search for inner disks in AGN was motivated by the observation of broad iron lines in Seyfert galaxies, as described in the review of relativistic disk lines by Miller (2007) and confirmed by recent observations with Chandra, XMM-Newton and Suzaku, and also discussed in the analysis of spectra by Nandra et al. (2007). These emission lines are the most obvious reaction of an accretion disk to irradiation by an external source of hard X-rays, which is true whenever a corona lies above a disk. The first clear example of relativistic dynamics affecting the line shape, that is a line originating in the innermost region, was found in the Seyfert 1 AGN MCG-6-30-15 by Tanaka et al. (1995), at that time using ASCA/SIS.

Miller (2007) compares how frequent detections of relativistic disk lines in LMXBs and Seyfert 1 AGN are: (1) in 75-85\% of the stellar sources relativistic disk lines were found. (2) For the sample of AGN with broad iron emission lines chosen by Nandra et al. (2007) the authors found that $45 \%$ of the sources are best fit with a relativistically blurred reflection model. But the comparison is difficult. For LMXBs inner weak disks were preferentially observed after the soft/hard transition, during a phase of accretion rate decrease, an only transient phenomenon. That phase lasts only a short time of the full outburst cycles, including quiescence. The detections in AGN refers to the sample of sources selected because of their iron line emission.

\subsection{The accretion rates of the best candidates}

We take the information from two samples of Seyfert galaxies. Group (1) are sources with "very strong relativistic disk line detections", Tier 1 of the census of Miller (2007). Group (2) are sources with "acceptable model fits including a broad Gaussian emission line component or a relativistically blurred line added to the spectrum" from the analysis of Nandra et al. (2007). We have not tried to exclude sources that might be in a high/soft spectral state.

Considering our sample of sources some special features need be commented. For some sources in group (2) differing results were found in multiple observations. The authors argued that non-detection of broad emission lines may be due to a low signal-to-noise ratio. The Nandra sample contains most of the sources in Millers Tier 1, his best candidates, but the judgment on the emission lines is not always the same: for NGC 3783 and MCG-6-30-15 the model fits of Nandra et al. were classified as poor. We kept these sources in our chosen sample. Despite its poor fit we included Ark 120 (sample of Nandra) in our sample because it is in Tier 3 of Miller (sources with detections that need to be confirmed and investigated more deeply). Because of heavy contamination with light from the galaxy (according to Vasudevan \& Fabian 2007), IC 4329A was not included.

For some sources a different X-ray luminosity is found from observations at different time, e.g. for NGC 4151 a change in 
$L_{\mathrm{X}}$ from $10^{42}$ to about $10^{42.8} \mathrm{erg} / \mathrm{s}$ between December 2000 and May 2003. For this observation in May 2003 from spectral fits Nandra et al. (2007) derived the value $10^{42.8} \mathrm{erg} / \mathrm{s}$, Vasudevan \& Fabian (2009) the value $10^{42.64} \mathrm{erg} / \mathrm{s}$. For NGC 3783 the luminosity found from observations on 2001 December 19 is $50 \%$ higher than that of December 17 (the difference maybe caused by an outflowing warm absorber). As the derivation of luminosity values for the same observations by different authors shows, the results of spectral fits occasionally differ by about $30 \%$ (in NGC 3783 and NGC 3516) and even more for NGC 4051 as observed in May 2001.

The samples of Miller (2007) and Nandra et al. (2007) were also used in a recent analysis of Brenneman \& Reynolds (2009) who selected eight candidates to investigate the relativistic broadening of iron emission lines for spin diagnostics. In half of the sources they found an improvement in their goodness-offit parameters when relativistic effects from an inner disk were taken into account.

The sources we finally consider are 3C 120, NGC 3516, NGC 3783, NGC 4051, NGC 4151, Mrk 766, and MCG-630-15 from Miller (2007) and in addition Mrk 590, Mrk 110, NGC 4395, NGC 5506, NGC 5548, Mrk 509, Ark 564, NGC 7469, and Ark 120 from Nandra et al. (2007). To clarify whether the broad iron emission lines of the sources in this sample could originate in a weak inner accretion disk that is surrounded by the ADAF carrying the main part of the accretion flow, we determine $L_{\text {bol }} / L_{\text {Edd }}$ and take this ratio as an approximate measure for the scaled accretion rate $\dot{M} / \dot{M}_{\text {Edd }}$.

For this evaluation the bolometric luminosities (from X-ray luminosities and X-ray bolometric corrections) and reliable black hole masses are needed. Recently Vasudevan \& Fabian (2007, 2009) determined X-ray bolometric corrections using spectral energy distributions (SED) in different wavebands (possible errors are between 10 and $100 \%$ in the first work, but only 1 to $10 \%$ in the second). We take the values for X-ray luminosity, black hole mass, bolometric correction and $L_{\mathrm{bol}} / L_{\mathrm{Edd}}$ from these investigations (where available the newer values). For sources not included in this investigation we take the X-ray luminosity from Nandra et al. (2007), together with a constant bolometric correction as used by Ho (2009a), and the black hole mass from O'Neill et al. (2005). For a few sources in our sample X-ray luminosities are also listed in the investigation of Ho et al. (2009b), estimates differ by up to a factor of three (even larger for NGC 4051). In Table 1 we list the derived values. For 10 sources the Eddington-scaled accretion rates are $\leq 0.1$. This value can be considered an upper limit for the transition between hard and soft spectral state). (Because the theoretically derived transition luminosity depends on parameters whose exact values are not determined by the simple modeling, but have to be assumed, one has to resort to observations for estimating the critical values; see also the values for the transition luminosity derived by Gierliński \& Newton 2006). For six sources rates clearly above 0.1 are found (possibly erroneously high values, see error bars).

The uncertainty of black hole masses affects the values of the Eddington-scaled luminosity. The values of black hole masses in the literature (also in Vasudevan \& Fabian 2007, 2009) largely go back to the compilation of Peterson et al. (2004), based on broad emission-line reverberation-mapping data with a typical uncertainty of $33 \%$. Comparing with black hole masses evaluated by other methods, e.g. the often used relation between black hole mass and central stellar velocity dispersion, a similar uncertainty is found. Nikołajuk et al. (2006) compared black hole masses determined from reverberation and the X-ray

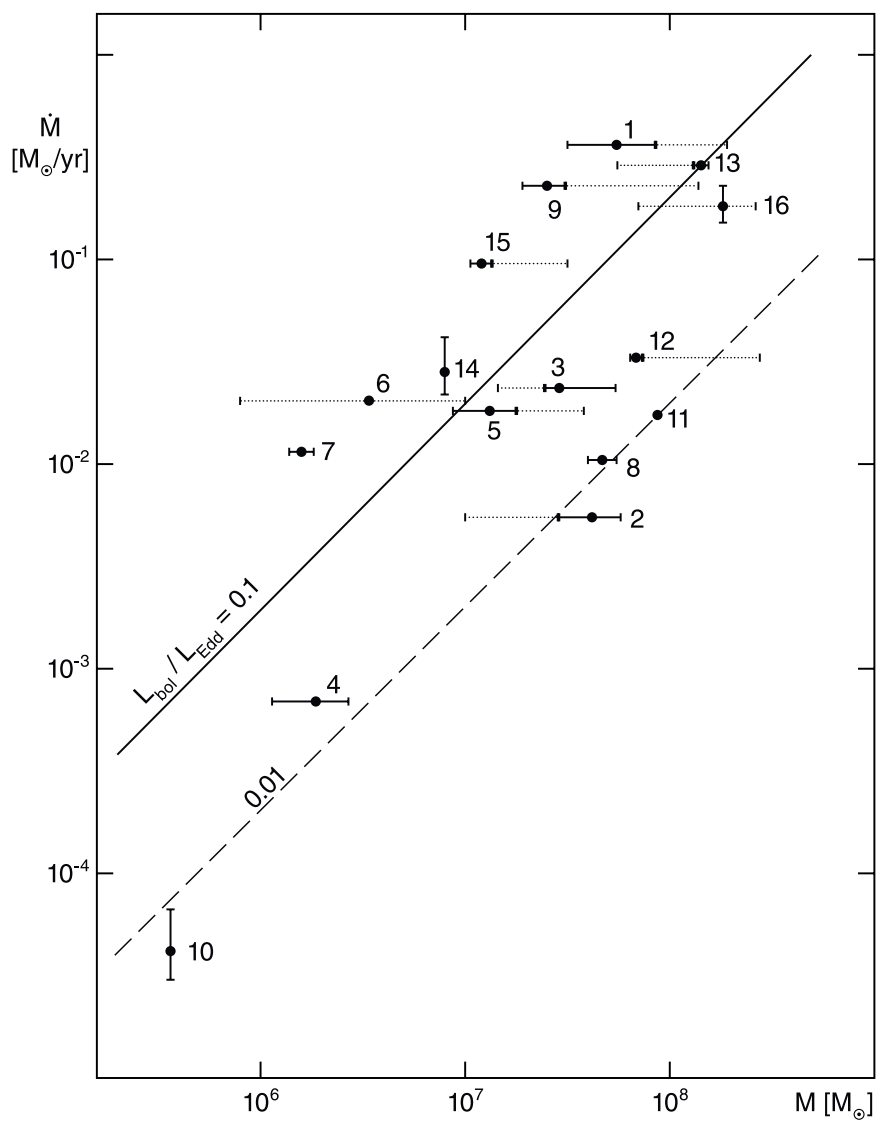

Fig. 2. Accretion rates of Seyfert galaxies with strong iron emission lines from inner accretion disks revealed in deep Chandra, XMM-Newton, and Suzaku observations (Miller 2007; Nandra et al. 2007); numbers in Table 1. Solid line: critical accretion rate corresponding to the soft/hard state transition; long-dashed line: 1/10 of critical rate; theory predicts the possible existence of an inner disk for accretion rates between these two lines. Horizontal error bars, solid lines: from Peterson et al. (2004), Nandra (2006); dotted lines: from Nikołajuk et al. (2006), Iwasawa et al. (2004), Markowitz et al. (2007), Boller et al. (2007); vertical error bars for $2-10 \mathrm{keV}$ luminosities and bolometric corrections given for sources 10,14 and 16 (Vasudevan \& Fabian 2007); not indicated for sources 1-5, 8, 9, 12, 13, and 15 (Vasudevan \& Fabian 2009) because the errors in the $2-10 \mathrm{keV}$ luminosities are small (see their Table 2), errors in bolometric corrections only a few percent. But see Sect. 5.2 for the general uncertainty of unscaled X-ray luminosities.

excess variance method and found large differences. For sources included in the recent catalog of central stellar velocity dispersions of nearby galaxies (Ho et al. 2009b), the black hole masses evaluated from the velocity dispersion values using the relation determined by Tremaine (2002) differ by a factor of $\leq 3$.

\subsection{Interpretation of the accretion rates}

For Seyfert galaxies NGC 3516, NGC 3783, NGC 4051, NGC 4151, Mrk 590, Ark 120, NGC 4395, NGC 4593, NGC 5506, and NGC 5548 the Eddington-scaled accretion rates are below 0.1 . This can be theoretically interpreted as a low spectral state, with an ADAF in the innermost region. The disk from which the emission lines come should then be a weak inner disk, separated by a gap from the outer truncated accretion disk, an accretion flow geometry similar to that in LMXBs.

In Fig. 2 we show the distribution of obtained accretion rates, given in $M_{\odot} /$ yr. The bolometric luminosities vary from $4 \times 10^{-5}$ 
to $0.367 M_{\odot} /$ yr. Error bars owing to uncertainty in black hole mass are widely given in the literature and are indicated in our figure. Errors in unscaled X-ray luminosity are difficult to judge (compare our discussion in Sect. 5.2). According to Vasudevan and Fabian (2009, Table 2) these errors are small. Error bars owing to uncertainty in bolometric correction are small for the sources examined in the work of Vasudevan \& Fabian (2009), a few percent only.

For six sources rates above 0.1 are found. This might be owing to errors because uncertainties enter in several ways. In the picture of a thin disk reaching all the way inward or an inner ADAF plus a truncated outer disk, depending on the accretion rate, one expects that these sources are in a standard high/soft spectral state with an untruncated disk reaching inward to the ISCO. But the photon index $\Gamma$ listed by Vasudevan \& Fabian (2009, Table 2) for these sources is $\leq 1.95$, or even lower, $\leq 1.5$. (For X-ray binaries Remillard \& McClintock (2006) chose $1.4 \leq \Gamma \leq 2.1$ for their definition of the low/hard state.) One of these sources, MCG-6-30-15, was classified as in high/soft state based on the comparison of the PSD (Uttley \& McHardy 2005), but the same was found for NGC 4051, for which a low accretion rate (scaled to the Eddington rate) was found. These examples show that it is more difficult to confirm the spectral state for AGN than for LMXBs.

For LMXBs an interesting observation is that e.g. GX 339-4 at one time was found in low/hard spectral state and at another time in the very high state with an extremely skewed relativistic Fe K $\alpha$ emission line (Miller et al. 2004).

It is conspicuous that in the distribution of accretion rates of the systems with $\mathrm{Fe} \mathrm{K} \alpha$ emission lines a dichotomy between low and very high values is found for LMXBs, but not for Seyfert galaxies with the same reflection features. For Seyfert galaxies we find sources with these rates in between the low and very high rates of LMXBs. Theory would predict continuous accretion disks inward toward the ISCO for these sources. If irradiation seems to be present this would mean that the innermost coronae in these AGN would be significantly stronger than those in LMXBs at comparable accretion rates. What could cause these differences between AGN and LMXBs? LMXBs are accreting binary systems, AGN accrete gas that has fallen in at large distances. That could in principle make a difference in the accretion of magnetic flux through their accretion disks, and this flux is possibly closely related to coronal dissipation and jet formation. These are interesting and challenging questions, but more theoretical work and probably also more observations are needed to make progress.

\section{Broad iron emission lines from untruncated disks: the narrow-line Seyfert 1 galaxy 1H0707-495}

For 1H0707-495, a narrow-line Seyfert 1 galaxy (NLS1), broad iron $\mathrm{K}$ and $\mathrm{L}$ emission lines were recently found (Fabian et al. 2009). The bright Fe-L emission allows us to detect a reverberation lag of $30 \mathrm{~s}$ between the direct X-ray continuum and its reflection from matter falling into the black hole, indicating that we obtain information from matter within a gravitational radius.

The accretion rate is estimated to be (just) sub-Eddington. The observations point to an untruncated disk reaching inward to the ISCO and Comptonization of the irradiating X-rays by the disk (for a discussion of the very high state see Done et al. 2007). These observations prove that $\mathrm{Fe} \mathrm{K} \alpha$ emission lines can be present in quite different geometries, always from disks in the innermost region, but possibly of two different kinds, weak inner disks in the hard spectral state or strong untruncated disks in a very high state. Spectral energy distributions from contemporaneous optical, UV and X-ray observations are needed to distinguish between these spectral states.

Some of the Seyfert 1 galaxies investigated for their broad iron emission lines by Miller (2007) and Nandra et al. (2007) might have similarity with $1 \mathrm{H} 0707-495$, but are not as bright as this source. Actually Ark 564 is known as a narrow line Seyfert 1 galaxy (Arévalo et al. 2006).

\section{Conclusions}

For LMXBs broad iron emission lines found in an intermediate hard spectral state were interpreted as originating from a weak inner disk, which is left over from a disk reaching inward to the ISCO at earlier time. The accretion flow geometry in the thin disk+ADAF model scales with the black hole mass. We discussed whether such a model for the interpretation of iron emission lines is also applicable to Seyfert galaxies.

We used recent observations and interpretations of broad, partly relativistically blurred iron emission lines in Seyfert 1 AGN. We considered sources in a hard spectral state. For the best candidates in the samples of Miller (2007) and Nandra et al. (2007) we evaluated accretion rates (scaled to the Eddington accretion rate) to see whether these rates point to a hard spectral state. As a limiting Eddington-scaled upper accretion rate for sources in the hard spectral state we chose the value 0.1, taking into account the observational results for the state transition luminosities of LMXBs. It is difficult to judge the uncertainty of the accretion rates evaluated for the Seyfert galaxies. Therefore we cannot definitely determine the spectral state. As dicussed in Sect. 3, it is not possible to derive firm information on the spectral state from the photon index of the spectral energy distribution.

For the majority of the sources considered here we found accretion rates $\leq 0.1$ (see Table 1), agreeing with the theoretical expectation of a weak inner accretion disk below the ADAF during the low/hard state for AGN as well. The appearance of iron $\mathrm{K} \alpha$ lines signifies the presence of cool material close to the accretng black hole, irradiated by a power-law continuum from a corona above. In this state the inner disk carries only a fraction of the total accretion flow, the major part flows through the corona and provides the hard luminosity. This situation is desribed in the modeling of the "truncated disk + corona + re-condensed inner disk" geometry. Disk truncation is an inherent feature of disk evaporation that explains the appearance of the hot, optically thin flow for low accretion rates (and also the hysteresis in transition luminosity between soft and hard spectral states).

Besides the Seyferts with accretion rates below the critical value there is a significant number of sources in our sample $(40 \%)$ that show accretion rates above this limit, though not as high as in some LMXBs in high state with iron emission lines (a significant fraction of the Eddington value). These LMXB sources probably are related to the so-called "very high" state, where the disk material is illuminated by a strong corona on top of an inner disk (Done et al. 2007). In the LMXB case a clear gap exists in the accretion rate distribution between the two groups with low and high rates, no sources with rates slightly above 0.1 are known. The galactic sources XTE J1650-500 and GX $339-4$ were observed in such a very high state, and extremely skewed relativistic Fe $\mathrm{K} \alpha$ emission lines were detected (Miller et al. 2002a, Miller et al. 2004). Miller (2007) pointed out that the spectral and variability phenomena then closely resemble the 
Table 1. Accretion rates deduced for Seyfert 1 galaxies with broad iron emission lines.

\begin{tabular}{llllll}
\hline \hline source & $\log L_{\mathrm{X}}$ & $\log M_{\mathrm{BH}}$ & $\kappa_{2-10 \mathrm{keV}}$ & $\frac{L_{\mathrm{bol}}}{L_{\mathrm{Edd}}}$ & $\begin{array}{l}\dot{M} \\
{\left[M_{\odot} / y\right]}\end{array}$ \\
& & & & & {$[1]$} \\
& {$[2]$} & {$[3]$} & {$[4]$} & {$[5]$} \\
\hline (1) 3C 120 & 44.0 & 7.74 & 20.6 & $0.305 \times$ & 0.367 \\
(2) NGC 3516 & 42.3 & 7.63 & 15.3 & 0.006 & 0.006 \\
(3) NGC 3783 & 42.9 & 7.47 & 17.3 & 0.036 & 0.023 \\
(4) NGC 4051 & 40.8 & 6.28 & 67. & 0.016 & 0.001 \\
(5) NGC 4151 & 42.8 & 7.12 & 17.4 & 0.062 & 0.002 \\
(6) Mrk 766 & 42.88 & 6.54 & 15.8 & $0.275 \times$ & 0.021 \\
(7) MCG-6-30-15 & 42.65 & 6.19 & 15.8 & $0.362 \times$ & 0.012 \\
(8) Mrk 590 & 43.0 & 7.68 & 7.0 & 0.010 & 0.011 \\
(9) Mrk 110 & 43.9 & 7.40 & 18.4 & $0.433 \times$ & 0.238 \\
(10) NGC 4395 & 40.02 & 5.56 & 22.5 & 0.005 & $410^{-5}$ \\
(11) NGC 5506 & 42.80 & 7.94 & 15.8 & 0.009 & 0.017 \\
(12) NGC 5548 & 43.3 & 7.83 & 10.1 & 0.024 & 0.035 \\
(13) Mrk 509 & 44.0 & 8.16 & 16.2 & 0.095 & 0.301 \\
(14) Ark 564 & 43.25 & 6.90 & 9.2 & $0.162 \times$ & 0.028 \\
(15) NGC 7469 & 43.2 & 7.09 & 42. & $0.369 \times$ & 0.099 \\
(16) Ark 120 & 43.82 & 8.27 & 15.7 & 0.044 & 0.180 \\
\hline
\end{tabular}

Notes. [1] $L_{\mathrm{X}} 2-10 \mathrm{keV}$ luminosity in units of erg/s, [2] $M_{\mathrm{BH}}$ black hole mass in units of $M_{\odot}$, [3] bolometric correction $\kappa_{2-10 \mathrm{keV}}=L_{\text {bol }} / L_{\mathrm{X}}$, [4] $L_{\mathrm{bol}} / L_{\mathrm{Edd}},[5] \dot{M}$ in $M_{\odot} / \mathrm{yr}, \dot{M} / \dot{M}_{\mathrm{Edd}} \approx L_{\mathrm{bol}} / L_{\mathrm{Edd}}$.

References. for sources 1-5, 8, 9, 12, 13, and 15 Cols. (1) to (4) from Vasudevan \& Fabian (2009); for sources 10, 14, and 16 Cols. (1) to (3) from Vasudevan \& Fabian (2007), for sources 6, 7, and $11 L_{X}$ from Nandra et al. (2007), $M_{\mathrm{BH}}$ from O'Neill et al. (2005), assumed constant bolometric correction 15.8 (Ho 2009a); crosses mark sources with an Eddington-scaled mass flow rate $\geq 0.1$.

behavior seen in Seyfert AGN. The narrow-line Seyfert 1 galaxy 1H0707-495 seems to be a particularly bright source of this kind, with an accretion rate just below the Eddington rate.

The Seyfert galaxies with accretion rates somewhat about the critical value (with no analogous stellar sources) deserve particular attension because the existence of these sources might indicate a difference between the accretion flow geometry around stellar mass and supermassive black holes. Theory predicts an untruncated disk for sources with accretion rates above 0.1 . The reflection features then indicate the presence of significantly stronger coronae at comparable accretion rates in these AGN than in LMXBs. If strong magnetic flux is the cause of this phenomenon, these coronae might well also be the base of a jet issuing from them. Such differences between accretion in stellar mass and supermassive black holes certainly deserve further work.

Weak inner disks below an ADAF-type accretion flow in the inner region of an accreting black hole can be considered as the natural remainder of an originally standard Shakura-Sunyaev disk that had reached inward all the way to the ISCO at high accretion rates and has now, when the accretion rate has dropped below its critical value, become truncated. This could therefore be a hint to a higher accretion rate in the past. Moderate accretion rate variations caused by the ionization instability in AGN disks could well allow the inner disk to exist for long times (as in the case of the galactic black hole binary Cyg X-1). Seyfert 1 galaxies with $10^{7}$ to $10^{8} M_{\odot}$ black holes and accretion rates of about 0.01 to $0.1 M_{\odot} / \mathrm{yr}$ seem specially suited to display this weak inner disk accretion flow geometry.
Acknowledgements. For helpful discussions and suggestions we thank Bifang Liu.

\section{Appendix A: The ionization instability in disks around stellar mass and supermassive black holes}

Accretion disks are thermally and viscously unstable at radii where the central temperature lies between about a few times $10^{3} \mathrm{~K}$ and $10^{4} \mathrm{~K}$. As a result the disk structure alternates between a hot, ionized structure and a cool non-ionized structure, corresponding to a high (outburst) or low (quiescence) mass flow rate in the disk. This instability is the cause of the dwarf nova outbursts (Meyer \& Meyer-Hofmeister 1981; Smak 1984, for a review of model calculations see Cannizzo 1993). The ionization instability is also the cause of the outbursts of soft X-ray transients (Dubus et al. 2001, and references therein).

The application of the disk instability model to AGN (Lin \& Shields 1986; Cannizzo 1992; Siemiginowska et al. 1996) is important in the framework of our analysis.

In their detailed modeling of the thermal-viscous ionization instability in AGN disks, Siemiginowska et al. (1996) had calculated the cyclic luminosity variation. Menou \& Quataert (2001) found that in contrast to the situation in dwarf novae and soft $\mathrm{X}$-ray transients, the gas remains well coupled to the magnetic field even on the cold branch of the limit cycle and consequently the parameter describing the viscosity should remain the same in hot and cold state. Siemiginowska et al. (1996) had evaluated the lightcurves for this case as well. Then the luminosity changes during the outburst cycle are not large, rather a "flickering" appears. In Fig. A.1 we show the effective temperature in disks around a supermassive black hole of $10^{7}, 10^{8}$ or $10^{9}$ solar masses for a variety of mass accretion rates $\dot{M}$ as a function of the distance $R$

$$
T_{\mathrm{eff}}=\frac{3}{8 \pi \sigma} \frac{G M \dot{M}}{R^{3}}\left(1-\sqrt{R_{\mathrm{ISCO}} / R}\right),
$$

with $G$ the gravitational constant, $M$ the mass of black hole, $\sigma$ the Stefan-Boltzman constant, $R_{\mathrm{ISCO}}$ the distance of the innermost stable orbit $=3 R_{\mathrm{S}}$ (non-rotating black hole), $R_{\mathrm{S}}=2 G M / c^{2}$ the Schwarzschild radius, and $c$ the velocity of light. The occurrence of the ionization instability depends on the midplane temperatures, which are typically double the effective temperature. To show the zones affected by the ionization instability in AGN we take the results from the investigation of Siemiginowska et al. (1996, Fig. 1): $3.1 \leq \log T_{\text {eff }} \leq 3.6$ (same viscosity parameter in hot and cold state, otherwise the instability strip reaches down to lower temperatures).

Menou \& Quataert (2001) analyzed the interplay between the various instabilities in disks around supermassive black holes. They pointed out that in low-luminosity AGN with $\dot{M} \leq$ $10^{-2} M_{\odot} /$ yr the disk is globally gravitationally stable, but in luminous AGN with $\dot{M} \geq 10^{-2} M_{\odot} /$ yr the disk can be globally gravitationally unstable except at small radii. We are interested in disks with moderate accretion rates and limit our investigation to mass flow variations caused by the ionization instability.

\section{Appendix B: A new aspect of AGN disk instabilities as a consequence of disk truncation}

Disk truncation as an additional feature in the accretion flow geometry can eliminate the occurrence of the ionization instability. 


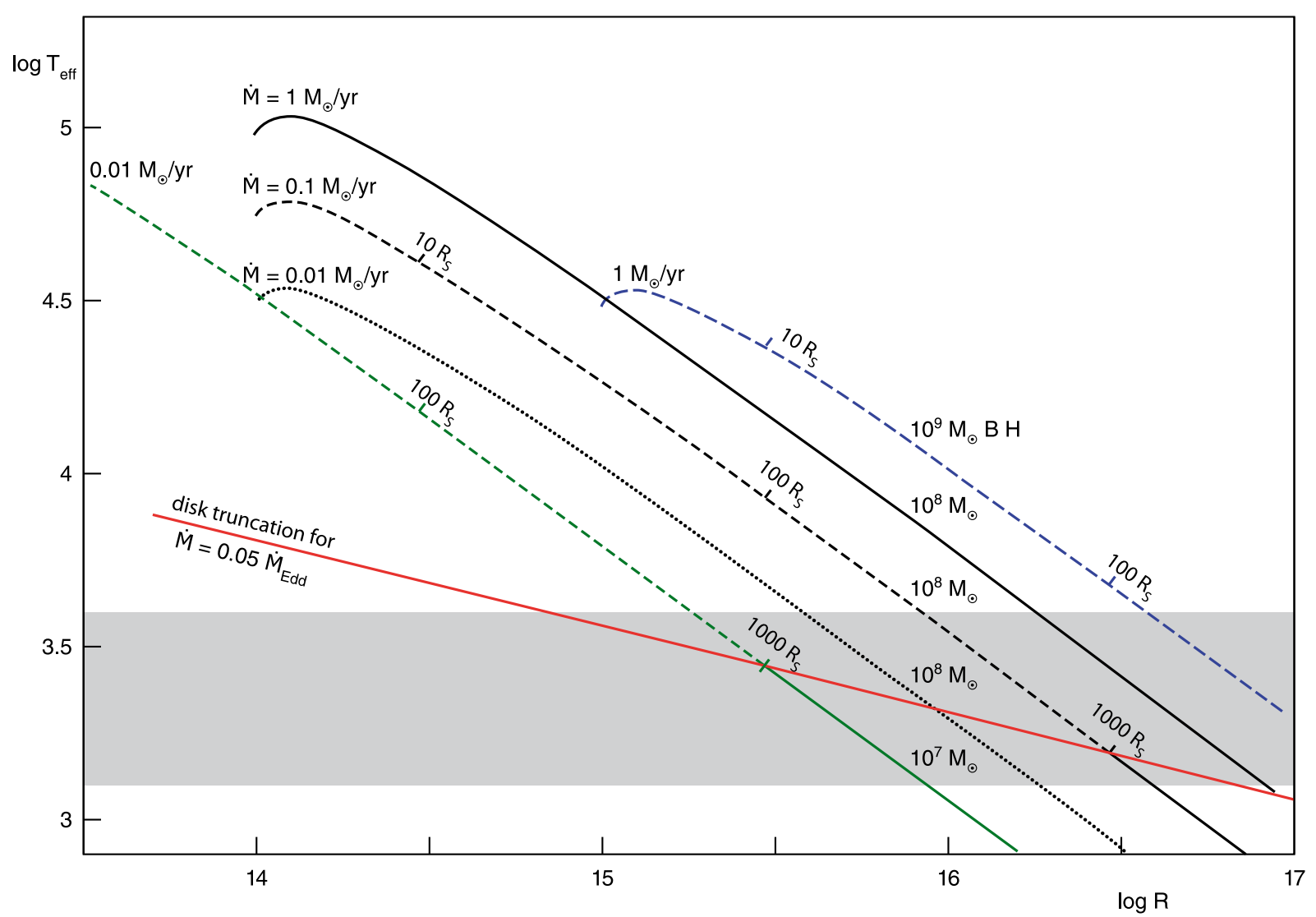

Fig. A.1. Effective temperatures of the disk, ionization instability strip (grey area), and disk truncation (inside ADAF). Truncation depends on the accretion rate (compare Fig. 1), in the figure solid lines for thin disk, dashed and dotted lines for ADAF; situation for a $10^{8} M_{\odot} \mathrm{BH}$ : with $\dot{M}=1 M_{\odot} /$ yr disk reaches inward to the last stable orbit (solid line); for $0.01 M_{\odot} / \mathrm{yr}$ the disk is truncated far out, beyond the range shown, inside ADAF only (dotted line); in between these two rates lies the critical accretion rate for spectral transition, of interest here, $0.1 M_{\odot} / \mathrm{yr}=0.05 \dot{M}_{\mathrm{Edd}}$ with disk truncation at about $1000 R_{\mathrm{S}}\left(R_{\mathrm{S}}\right.$ Schwarzschild radius) (dashed line/solid line); for $10^{9} M_{\odot}$ and $10^{7} M_{\odot}$ BHs: only curves for the critical accretion rate are shown.

Evaporation (discussed in Sect. 2) is expected to lead to the disappearance of inner regions, if the accretion rate in Eddington units lies below the critical value $\dot{m}_{\text {crit }}$. The truncation then occurs at a distance of $R \geq 300 R_{\mathrm{S}}$. For lower accretion rates the truncation might be far out, at thousands of Schwarzschild radii, e.g. in low-luminosity AGN (see Narayan 2005, Fig. 3).

In Fig. A.1 we show the situation for different black hole masses and different accretion rates. For a $10^{8} M_{\odot}$ black hole: (1) for a high accretion rate $\dot{M}=1 M_{\odot} /$ yr the disk reaches inward to the ISCO; (2) for a low accretion rate $\dot{M}=10^{-2} M_{\odot} / \mathrm{yr}$ the disk is truncated at about $10^{17.1} \mathrm{~cm}$ (Meyer-Hofmeister \& Meyer 2006), far outside the instability strip; (3) for the rate $\dot{M}=10^{-1} M_{\odot} / \mathrm{yr} \approx \dot{m}_{\text {crit }} \approx 0.045$, which is the critical rate for the spectral state transition where we expect a disk truncation at a distance of about $1000 R_{S}$, lying within the instability strip. For $10^{7} M_{\odot}$ and $10^{9} M_{\odot}$ black holes the temperature distribution is only shown for the critical accretion rate.

As a consequence of disk truncation the unstable regions are partly eliminated, as can be seen from Fig. A.1. For instance an unstable region as described by Menou \& Quataert (2001, Fig. 1) could not be present at the distance of $30 R_{\mathrm{S}}$ from a $10^{7} \mathrm{M}_{\odot}$ black hole.

For the question whether a weak inner disk could be expected (accretion rate close to the critical rate for state transition, dashed lines in Fig. A.1), two conclusions can be drawn: (1) the "flickering" (instead of large luminosity variation) supports the existence of an inner disk if the accretion rate is close to the critical one for state transition; (2) for these rates (dashed lines in Fig. A.1) for black hole masses of $10^{7} M_{\odot}$ to $10^{8} M_{\odot}$ the disk instability can be present, for higher masses it may be eliminated, that is, the mass flow can be stable.

For large black holes of $10^{9} M_{\odot}$ the ionization instability may occur as a special feature in a weak inner accretion disks, if the temperature there does not allow a stable structure.

\section{References}

Abramowicz, A., Chen, X., Kato, S., et al. 1995, ApJ, 438, L37 Arévalo, P., Papadakis, I. E., \& Uttley, P. 2006, MNRAS, 372, 401 Boller, Th., Balestra, I., \& Kollatschny, W. 2007, A\&A, 465, 87 Brenneman, L. W., \& Reynolds, C. S. 2009, ApJ, 702, 1367

Cabanac, C., Fender, R. P., Dunn, R. J. H., et al. 2009, MNRAS, 396, 1415 Cannizzo, J. K. 1992, ApJ, 385, 94

Cannizzo, J. K. 1993, in Accretion Discs in Compact Stellar Systems, ed. J. C. Wheller, 6

Chen, W., Shrader, C. R., \& Livio, M. 1997, ApJ, 491, 312

Di Matteo, T., Quataert, E., Allen, S. W., et al. 2000, MNRAS, 311, 507 Di Matteo, T., Allen, S. W., Fabian, A. C., et al. 2003, ApJ, 582, 133 Done, C., \& Gierlińsli, M. 2005, Astrophys. Space Sci., 300, 167

Done, C., Gierlińsli, M., \& Kubota, A. 2007, A\&ARv, 15, 1 Dubus, G., Hameury, J.-M., \& Lasota, J.-P. 2001, A\&A, 373, 251 Dunn, R. J. H., Fender, R. P., \& Körding, 2010, MNRAS, 403, 61 Esin, A. A., McClintock, J. E., \& Narayan, R. 1997, ApJ, 489 Esin, A. A., Narayan, R., Cui, W., et al. 1998, ApJ, 505, 854 Fabian, A. C., Zoghbi, A., Ross, R. R., et al. 2009, MNRAS, 392, 1124 Falcke, H., \& Biermann, P. L. 1999, A\&A, 342, 49 
Falcke, H., \& Markoff, S. 2000, A\&A, 362, 113

Falcke, H., Nagar, N. M., Wilson, A. S., et al. 2000, ApJ, 542, 197

Gierliński, M., \& Newton, J. 2006, MNRAS, 370, 837

Hiemstra, B., Mendez, M., Done, C., et al. 2011, MNRAS, 411, 137

Ho, L. C. 2009a, ApJ, 699, 626

Ho, L. C., Greene, J. E., Fillipenko, A. V., et al. 2009b, ApJS, 183, 1 Iwasawa, K., Miniutti, G., \& Fabian, A. C. 2004, MNRAS, 355, 1073 Lin, D. N. C., \& Shields, G. A. 1986, ApJ, 305, 28

Liu, B. F., \& Meyer-Hofmeister, E. 2001, A\&A, 372, 386

Liu, B. F., Mineshige, S., Meyer F., et al. 2002, ApJ, 575, 117

Liu, B. F., Meyer, F., \& Meyer-Hofmeister, E. 2006, A\&A, 442, 555

Liu, B. F., Taam, R. E., Meyer-Hofmeister, E., et al. 2007, ApJ, 671, 695

Liu, B. F., \& Taam, R. E. 2009, ApJ, 707, 233

Markowitz, A., Edelson, R., Vaughan, S., et al. 2003, ApJ, 593, 96

Markowitz, A., Papadakis, I., \& Arévalo, P. 2007, ApJ, 656, 116

McClintock, J. E., \& Remillard, R. A. 2006, in Compact Stellar X-ray Sources, ed. W. H. G. Lewin, \& M. van der Klis (Cambridge University Press), Cambridge Astrophysics Series, No. 39, 157

McHardy, I. M. 2009, in The Jet Paradigm - From Microquasars to Quasars, ed. T. Belloni, Lect. Notes Phys., 794, 203

McHardy, I. M., Uttley, P., \& Vaughan, S., 2005, MNRAS, 359, 345

Menou, K., \& Quataert, E. 2001, ApJ, 552, 204

Meyer, F., \& Meyer-Hofmeister, E. 1981, A\&A, 104, L10

Meyer, F., \& Meyer-Hofmeister, E. 1994, A\&A, 288, 175

Meyer, F., Liu, B. F., \& Meyer-Hofmeister, E. 2000, A\&A, 354, L67

Meyer, F., Liu, B. F., \& Meyer-Hofmeister, E. 2007, A\&A, 463, 1

Meyer-Hofmeister, E., \& Meyer, F. 2006, A\&A, 449, 443

Meyer-Hofmeister, E., Liu, B. F., \& Meyer, F. 2005, A\&A, 432, 181

Meyer-Hofmeister, E., Liu, B. F., \& Meyer, F. 2009, A\&A, 508, 329

Miller, J. M., 2007, ARA\&A, 45, 441

Miller, J. M., Fabian, A. C., Wijnands, R., et al. 2002a, ApJ, 570, L69

Miller, J. M., Fabian, A. C., in 't Zand, J. J. M. et al. 2002b, ApJ, 577, L15
Miller, J. M., Fabian, A. C., Reynolds, C. S., et al. 2004, ApJ, 606, L131

Miller, J. M., Homan, J., \& Miniutti, G. 2006a, ApJ, 652, L113

Miller, J. M., Homan, J., Steeghs, D., et al. 2006b, ApJ, 653, 525

Miller, L., Turner, T. J., \& Reeves, J. N. 2008, A\&A, 483, 437

Nandra, K. 2006, MNRAS, 368, L62

Nandra, K., O’Neill, P. M., George, I. M., \& Reeves, J. N. 2007, MNRAS, 382 , 194

Narayan, R. 2005, Astrophys. Space Sci., 300, 177

Narayan, R., \& Yi, I. 1994, ApJ, 428, L13

Narayan, R., \& Yi, I. 1995, ApJ, 452, 710

Narayan, R., \& McClintock, J. E. 2008, New Astron. Rev., 51, 733

Narayan, R., Mahadevan, R., \& Quataert, E. 1998, in Theory of Black Hole Accretion Disks, ed. M. A. Abramowicz, G. Bjornson, \& J. E. Pringle (Cambridge Univ. Press), 148

Nikołajuk, M., Czerny, B., Ziółkowski, J., et al. 2006, MNRAS, 370, 1534

O’Neill, P. M., Kirpal, N., Papadakis, I. E., et al. 2005, MNRAS, 358, 1405

Peterson, B. M., Ferrarese, L., Gilbert, K. M., et al. 2004 ApJ, 613, 682

Qiao, E., \& Liu, B. F. 2010, PASJ, 62, 661

Reis, R. C., Fabian, A. C., Ross, R., et al. 2008, MNRAS, 392, 992

Reis, R. C., Fabian, A. C., \& Miller, J. M. 2010, MNRAS, 402, 836

Remillard, R. A., \& McClintock, J. E. 2006, ARA\&A, 44, 49

Rykoff, E. S., Miller, J. M., Steeghs, D., et al. 2007, ApJ, 666,1129

Shemmer, O., Brandt, W. N., Netzer, H., et al. 2006, ApJ, 646, L29

Siemiginowska, A., Czerny, B., \& Kostyunin, V. 1996, ApJ, 458, 491

Smak, J. 1984, Acta Astron., 34, 161

Taam, R. E., Liu, B. F., Meyer, F., et al. 2008, ApJ, 688, 527

Tanaka, Y., Nandra, K., Fabian, A. C., et al. 1995, Nature, 375, 659

Tomsick, J. A., Kalemci, E., Kaaret, P., et al. 2008, ApJ, 680, 593

Tremaine, S., Gebhardt, K., Bender, R., et al. 2002, ApJ, 574, 740

Uttley, P., \& McHardy, I. M., 2005, MNRAS, 363, 586

Vasudevan, R. V., \& Fabian, A. C. 2007, MNRAS, 381, 1235

Vasudevan, R. V., \& Fabian, A. C. 2009, MNRAS, 392, 1124

Yuan, F., 2007, ASP Conf. Ser., 373, 95 